\title{
COULOMB BLOCKADE OF THE AHARONOV-BOHM EFFECT
}

\author{
C. W. J. Beenakker, H. van Houten, and A. A. M. Staring1 \\ Philips Research Laboratories \\ 5600 JA Eindhoven, The Netherlands
}

\section{INTRODUCTION}

Granular electronics, the theme of this conference, refers to conduction phenomena governed by the transport of a single quantum of charge $e$. The Aharonov-Bohm effect refers to oscillations in the conductance governed by the addition to the system of a single quantum of magnetic flux $h / e$. The present article addresses the interplay of these two quanta of nature.

The granularity of the transported charge manifests itself in the conductance as a result of the Coulomb repulsion of individual electrons. The transfer by tunneling of one electron between two initially neutral regions, of mutual capacitance $C$, increases the electrostatic energy of the system by an amount of $e^{2 / 2 C}$. At low temperatures and small applied voltages, conduction is suppressed because of the charging energy (Gorter, 1951). This phenomenon is now known as the Coulomb blockade of single-electron tunneling (Likharev, 1988; and in this volume).

The Aharonov-Bohm effect is a quantum interference effect which results from the influence of the vector potential on the phase of the electron wavefunction. Aharonov and Bohm $(1959,1961)$ originally considered the influence of the vector potential on electrons confined to a multiply-connected region, within which the magnetic field is zero. The ground state energy of the system is periodic in the enclosed flux with period $h / e$, as a consequence of gauge invariance (cf. the article by Leggett in this volume). Coulomb repulsion does not affect this periodicity.

In the solid state, the Aharonov-Bohm effect manifests itself as a periodic oscillation in the conductance of a sample as a function of an applied magnetic field $B$. A well-defined periodicity requires that the conducting paths through the sample enclose a constant area $A$, perpendicular to $B$. The periodicity of the oscillations is then $\Delta B=h / e A$, plus possibly harmonics (e.g. at $h / 2 e A$ ). The constant area may be imposed by confining the electrons electrostatically to a ring or to a cylindrical film (Washburn and Webb, 1986; Aronov and Sharvin, 1987). Alternatively, one can use the magnetic field itself to confine the Fermi-level electrons to the edge of a singly-connected region, thereby creating effectively a ring topology. The Aharonov-Bohm effect due to such circulating edge states was studied in metals in weak magnetic fields (Bogachek and Gogadze, 1973; Brandt et al., 1977), and more recently in semiconductors in strong magnetic fields in the quantum Hall effect regime (Van Loosdrecht $e t$ al., 1988; Van Wees et al., 1989; Sivan et al., 1989).

\footnotetext{
1 Also at: Eindhoven University of Technology, 5600 MB Eindhoven, The Netherlands.
} 
An essentidl difference with the original Aharonov-Bohm effect is that in these experiments the magnetic field extends into the conducting region of the sample Since the periodicity is now no longer constraned by gauge invariance, this opens up the possibility, in principle, of an influence of Coulomb repulsion In the present article we discuss our theoretical work on the suppression of the Aharonov-Bohm effect by the Coulomb blockade of tunneling, in more detail than in the original publication (Beenakker, Van Houten, and Staring, 1990) The suppression is predicted to occur in a "quantum dot", $1 \mathrm{e}$ a disc shaped region in a two dimensiondl electron gds, for a capacitance which is sufficiently small that the chatging energy $e^{2} / C$ becomes compardble to the separation of Landau levels $\hbar \omega_{c}$ (with $\omega_{c} \equiv e B / m$ the cyclotron frequency) A precursor at larger capacitances is a reduction of the frequency of the magnetoconductance oscillations, by a factor of $1+e^{2} / C \Delta E$ (with $\Delta E$ the energy separation of the edge states) The influence of the Coulomb repulsion disappears, in dccord with the original Aharonov Bohm effect, if a large hole is made in the quantum dot, such that the area of the conducting region $S$ of the resulting ring is much smaller than the enclosed area $A$

In the next section, we analyze in general terms the influence of Coulomb repulsion on resonant tunneling Some well known properties of circulating edge states in a quantum dot are reviewed in the subsequent section In the fourth section we then combine the results of the two preceding sections to obtain the suppression of the Aharonov-Bohm effect in a disc, and its recovery in a ing An experimental test of the theory is then suggested

\section{COULOMB OSCILLATIONS AND RESONANT TUNNELING}

To andlyze the influence of Coulomb repulsion on resonant tunneling we consider a quantum dot which is weakly coupled by tunnel barriers to two electron reservors The electiostatic potential profile along a line through the quantum dot is shown schematically in Fig 1 (discussed below) A current $I$ can be passed through the dot by applying a voltage difference $V$ between the two reservoirs The conductance $G$ of the quantum dot is defined as $G=I / V$, in the limit $V \rightarrow 0$ In the absence of Coulomb repulsion, the condition for $a$ conductance pedk due to resonant tunneling through the quantum dot is simply that the Ferm energy $E_{\Gamma}$ in the reservors lines up with an energy level in the dot We wish to know how that condition is modified by the charging energy
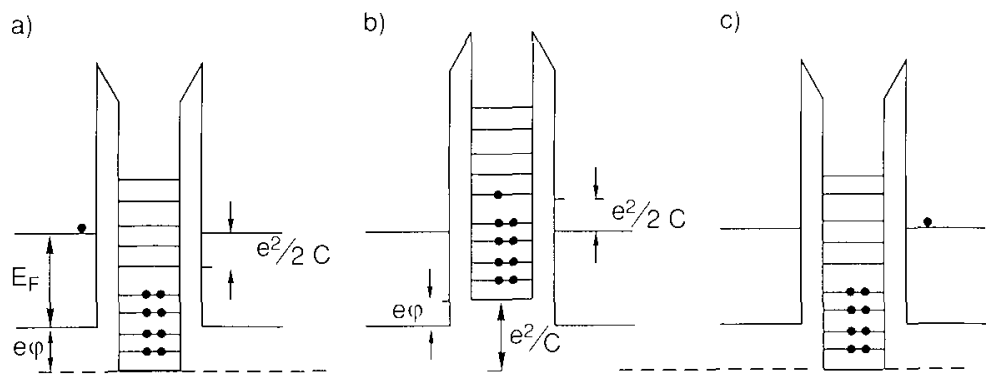

Fig 1 Single electron tunneling through a quantum dot, under the conditions of (6), for the case that the charging energy is comparable to the level spacing An infinitesimally small voltage difference is assumed between the left and nght reservours

The linear response conductance $G$ can be andlyzed with the equilibrium properties of the system Let us consider these The probability $P(N)$ to find $N$ electrons in the quantum dot in equilibrium with the reservoirs is given by the grand canonical distribution function

$$
P(N)=\text { constant } \times \exp \left(-\frac{1}{k T}\left[F(N)-N E_{\mathrm{F}}\right]\right),
$$

where $F(N)$ is the free energy of the dot and $T$ the temperature The reservorr Ferm energy $E_{\mathrm{F}}$ 
is measured relative to the conduction band bottom in the reservoirs. In general, $P(N)$ at $T=0$ is non-zero for a single value of $N$ only (namely the integer which minimizes the thermodynamic potential $\left.\Omega(N) \equiv F(N)-N E_{\mathrm{F}}\right)$. In that case, $G \rightarrow 0$ in the limit $T \rightarrow 0$. As discussed by Glazman and Shekhter (1989), a non-zero $G$ is possible only if $P(N)$ and $P(N+1)$ are both non-zero for some $N$. Then a small applied voltage is sufficient to induce a current through the dot, via intermediate states $N \rightarrow N+1 \rightarrow N \rightarrow N+1 \rightarrow \ldots$. To have $P(N)$ and $P(N+1)$ both non-zero at $\mathrm{T}=0$ requires that both $N$ and $N+1$ minimize $\Omega$. A necessary condition is $\Omega(N+1)=\Omega(N)$, or

$$
F(N+1)-(N+1) E_{\mathrm{F}}=F(N)-N E_{\mathrm{F}} .
$$

This condition is also sufficient, unless $Q$ has more than one minimum (which is usually not the case). At $T=0$ the free energy $F(N)$ equals the ground state energy $U(N)$ of the dot. We conclude that a peak in the low-temperature conductance occurs whenever

$$
U(N+1)-U(N)=E_{\mathrm{F}},
$$

for some integer $N$.

The usefulness of (3) is that it maps the problem of determining the location of the conductance peaks onto the more familiar problem of calculating the electrochemical potential $U(N+1)-U(N)$ of the quantum dot, i.e. the binding energy of one electron to the dot. This opens the way, in principle, to a study of exchange and correlation effects on conductance oscillations in a quantum dot [e.g. along the lines of work by Bryant (1987), and by Maksym and Chakraborty (1990)].

In the present investigation we adopt the simple approximation usually made in studies of the Coulomb blockade (Likharev, 1988; Glazman and Shekhter, 1989; Korotkov et al., 1990 , Averin and Korotkov) of taking the Coulomb interaction into account only via the macroscopic electrostatic energy $\int \phi(Q) d Q$. Here $Q=-N e$ is the charge on the dot, and

$$
\phi(Q)=-\frac{N e}{C}+\phi_{c \times t}
$$

is the potential difference between dot and reservoir, including a contribution $\phi_{\mathrm{cxt}}$ from external charges. ${ }^{2}$ We thus write for the ground state energy: ${ }^{3}$

$$
U(N)=\sum_{p=1}^{N} E_{p}+\frac{(N e)^{2}}{2 C}-N e \phi_{\mathrm{cxt}},
$$

where $E_{p}(p=1,2, \ldots)$ are the single-electron energy levels in ascending order, measured relative to the bottom of the potential well in the quantum dot. Each level contains either one or

2 In a two-dimensional clectron gas, the external charges are supplied by ionized donors and by a gate clectrode (with an electrosiatic voltage $V_{\text {gate }}$ between gate and reservoir). One has $\phi_{\text {ext }}=\phi_{\text {donors }}+\alpha V_{\text {gate, }}$ where $\alpha$ (as well as $C$ ) is a rational function of the capacitancc matrix clements of the system.

3 To make connection with some of the litcrature (Büttiker, 1987; Amman et al., 1989), we mention that $Q_{\text {ext }}$ $\equiv C \phi_{\mathrm{cxt}}$ plays the role of an "cxtcrnally induced charge" on the dot, which can be varicd continuously by mcans of $V_{\text {gatc }}$ (in contrast to $Q$ which is restricted to integer multiples of $e$ ). In terms of $Q_{\text {cxt }}$ one can writc

$$
U(N)=\sum_{p=1}^{N} E_{p}+\frac{\left(N e-Q_{\mathrm{ext}}\right)^{2}}{2 C}+\text { constant }
$$

which is cquivalent to (5). We emphaslze that $Q_{\mathrm{ext}}$ is an externally controlled variable, via $V_{\text {gate, }}$, regardless of the relative magnitude of the various capacitances in the system. 
zero electrons. Spin degeneracy can be included by counting each level twice, and other degeneracies can be included similarly. The energy levels $E_{p}$ depend on gate voltage and magnetic field, but are assumed to be independent of $N$. This assumption is supported by recent self-consistent solutions of the Schrödinger and Poisson equations in a quantum dot (Kumar et al., 1990).

$$
\text { Substitution of (5) into (3) gives (after relabeling } N+1 \rightarrow N \text { ) }
$$

$$
E_{N}^{*} \equiv E_{N}+\frac{(2 N-1) e^{2}}{2 C}=E_{F}+e \phi_{\mathrm{ext}}
$$

as the condition for a conductance peak. The left-hand-side of (6) defines a renormalized energy level $E_{N}^{*}$. The renormalized level spacing $\Delta E^{*}=\Delta E+e^{2} / C$ is enhanced above the bare level spacing by the charging energy. In the limit $e^{2} / C \Delta E \rightarrow 0,(6)$ is the usual condition for resonant tunneling. In the limit $e^{2} / C \Delta E \rightarrow \infty$, and for $B=0$, (6) describes the periodicity of the Coulomb oscillations in the conductance versus electron density (see below), studied theoretically in several papers (Amman et al., 1989; Glazman and Shekhter, 1989; Van Houten and Beenakker, 1989). The interplay of resonant tunneling and Coulomb oscillations at $B=0$ has been studied recently by Wingreen and Lee (1990), by means of a self-consistent solution of the Schrödinger and Poisson equations. Note that (6) is sufficient to determine the periodicity of the conductance oscillations, but gives no information on their amplitude and width. That requires the solution of a kinetic equation, with input of the tunneling rates. Such a calculation has been performed by Korotkov et al. (1990) for the non-linear I-V characteristic of a quantum dot at $B=0$.

In Fig. 1 we have illustrated the tunneling of an electron through the dot under the conditions of (6). In panel (a) one has $E_{N}+e^{2} / 2 C=E_{\mathrm{F}}+e \phi(N-1)$, with $N$ referring to the lowest unoccupied level in the dot. In panel (b) an electron has tunneled into the dot. One now has $E_{N}-e^{2} / 2 C=E_{\mathrm{F}}+e \phi(N)$, with $N$ referring to the highest occupied level. The potential difference $\phi$ between dot and reservoir has decreased by $e / C$ (becoming negative), because of the added electron. Finally, in panel (c) the added electron tunnels out of the dot, resetting the potentials to the initial state of panel (a).

The conductance of the quantum dot oscillates as a function of the Fermi energy (or electron density) of the reservoirs. The periodicity $\Delta E_{\mathrm{F}}$ follows from (6). If $E_{\mathrm{F}}$ is increased at constant $\phi_{\mathrm{ext}}$, one has simply

$$
\Delta E_{\mathrm{F}}=\Delta E^{*} \equiv \Delta E+\frac{e^{2}}{C}
$$

In the absence of charging effects, $\Delta E_{\mathrm{F}}$ is determined by the irregular spacing $\Delta E$ of the singleelectron levels in the quantum dot. The charging energy $e^{2} / C$ regulates the spacing, once $e^{2} / C$ $\gtrsim \Delta E$. The spin degeneracy of the levels is lifted by the charging energy. In a plot of $G$ versus $E_{\mathrm{F}}$ this leads to a doublet structure of the oscillations, with a spacing alternating between $e^{2} / C$ and $\Delta E+e^{2} / C$.

Experiments on conductance oscillations as a function of gate voltage have been analyzed in terms of (6) by Staring et al. (1990). In these, and related experiments (ScottThomas et al., 1989; Meirav et al., 1989; 1990; Kouwenhoven et al., 1990), both $E_{\mathrm{F}}$ and $\phi_{\mathrm{cxt}}$ are varied by changing the voltage on a gate electrode which defines a confined region in a twodimensional electron gas. In addition, a change in gate voltage affects the shape of the confining potential, and hence the single-electron levels $E_{p}$. The confined region in these experiments consists of a segment of a narrow channel, delimited by tunnel barriers. Under the realistic assumption of a linear dependence on the gate voltage of the number of electrons per unit channel length (Laux et al., 1988), it follows from (6) that the conductance oscillations remain approximately periodic in the gate voltage for such a geometry, thus explaining the most remarkable feature of the experiments. 


\section{CIRCULATING EDGE STATES}

Entirely new mechanisms for the Aharonov-Bohm effect become operative in strong magnetic ficlds in the quantum Hall effect regime. These mechanisms do not requirc a ring geometry, but apply to singly-connected geometries such as a point contact (Van Loosdrecht $e t$ al., 1988), or a quantum dot (Van Wees et al., 1989; Sivan et al., 1989). These geometries behave as if they were multiply connected, because of circulating edge states. In this section we review some well-known properties of edge states in a noninteracting electron gas, which we will need below. A comprehensive treatment of edge state transport can be found in a recent review (Beenakker and Van Houten, 1991).

In a strong perpendicular magnetic field $B$ and a smooth confining potential $V(r)$, the single-electron states of a two-dimensional electron gas are extended along equipotentials of $V$ at the guiding center energy $E_{\mathrm{G}}$, defined by

$$
E_{G_{3}}=E-\left(n-\frac{1}{2}\right) \hbar \omega_{c},
$$

for an electron with energy $E$ in the $n$th Landau level $(n=1,2, \ldots)$. The confining potential should be sufficiently smooth that it does not induce transitions between different values of $n$. This requires that $l_{\mathrm{m}} V^{\prime} \lesssim \hbar \omega_{\mathrm{c}}$, with $l_{\mathrm{m}} \equiv(\hbar / e B)^{1 / 2}$ the magnetic length (which plays the role of the wave length in the quantum Hall effect regime). The energy levels $E_{n p}$ for a given $n$ are such that the (closed) equipotentials for subsequent $p$ enclose one additional quantum of flux h/e.

A canonical example is the harmonic oscillator potential $V(r)=m \omega_{0}^{2} r^{2} / 2$, for which the single-electron Schrödinger equation can be solved exactly. The exact energy levels (for a single spin direction) are (Fock, 1928; Darwin, 1930)

$$
\begin{aligned}
E_{m l} & =\frac{1}{2} l \hbar \omega_{c}+\hbar\left(\omega_{c}^{2}+4 \omega_{0}^{2}\right)^{1 / 2}\left(m+\frac{|l|}{2}-\frac{1}{2}\right), \\
m & =1,2, \ldots, \quad l=0, \pm 1, \pm 2, \ldots .
\end{aligned}
$$

In the limit $\omega_{0} / \omega_{c} \rightarrow 0$ of a smooth potential, (9) reduces to

$$
E_{n s}=\hbar \omega_{\mathrm{c}}\left[n-\frac{1}{2}+s\left(\frac{\omega_{0}}{\omega_{c}}\right)^{2}\right], n=1,2, \ldots, \quad s=n, n+1, n+2, \ldots
$$

with the identifications $n=(l+|l|) / 2+m, s=2 m+|l|-1$. Equation (10) may also be written as

$$
\begin{gathered}
E_{n p}=\left(n-\frac{1}{2}\right) \hbar \omega_{\mathrm{c}}+V\left(R_{n p}\right), \quad B \pi R_{n p}^{2}=\left(p+\gamma_{n}\right) \frac{h}{e}, \\
n=1,2, \ldots, \quad p=1,2, \ldots,
\end{gathered}
$$

which is equivalent to the requirement that the equipotential of the edge state, of radius $R_{n p}$, encloses $p+\gamma_{n}$ flux quanta. Cornparison with (10) shows that, for the harmonic oscillator potential, $\gamma_{n}=n-1$. For other smooth confining potentials $V(r)$, (11) still holds, but $\gamma_{n}$ may be different. We will make use of (11) in the last section to obtain the approximate energy levels for a ring-shaped confining potential. (Knowledge of $\gamma_{n}$ is not important if one only considers states within a single Landau level.) 
Equation (11) does not hold for a hard-wall confining potential. An exact solution exists in this case for a circular disc of radius $R$, defined by $V(r)=0$ for $r<R$, and $V(r)=\infty$ for $r>R$ (Geerinckx et al., 1990). The case of a square-shaped disc was studied numerically by Sivan et al. $(1988 ; 1989)$. In Fig. 2 we show the energy spectrum as a function of $B$ for the latter case. The asymptotes correspond to the bulk Landau levels $E=(n-1 / 2) \hbar \omega_{c}$. The first two Landau levels $(n=1,2)$ are visible in Fig. 2 . The states between the Landau levels are edge states, which extend along the perimeter of the disc. These circulating edge states make the geometry effectively doubly connected - in the sense that they enclose a well-defined amount of flux. This is at the origin of the Aharonov-Bohm effect in a quantum dot.

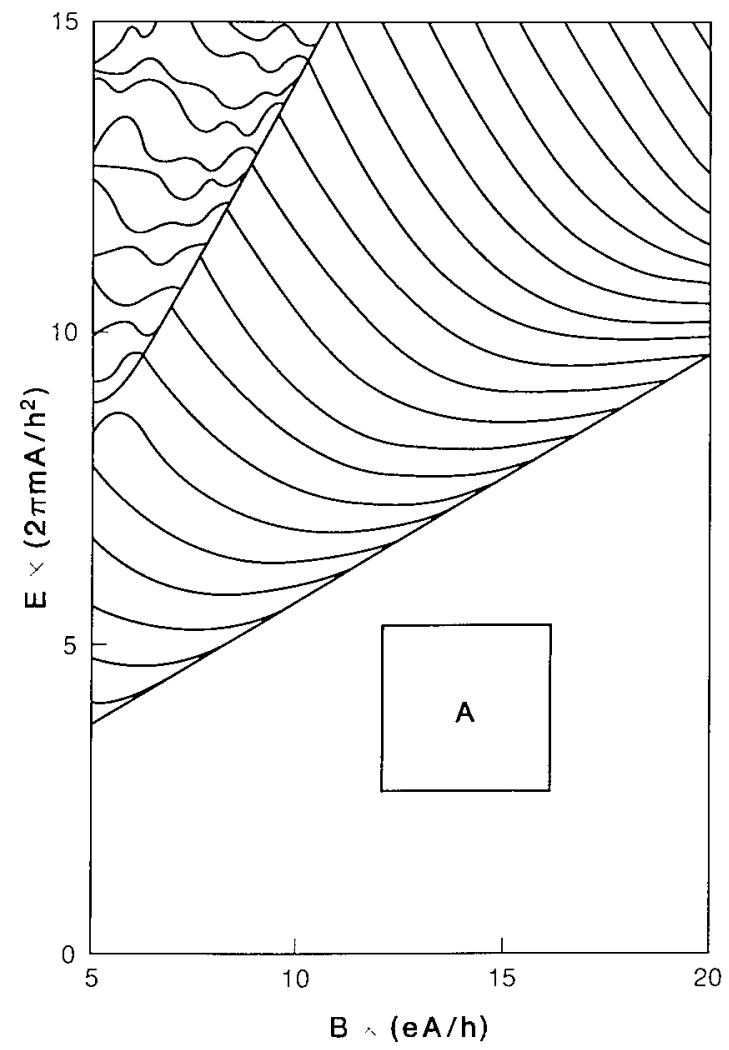

Fig. 2 Energy levels as a function of magnetic field for a square-shaped disc (of area $A$ ) defined by a hard-wall confining potential, as calculated by Sivan et al. (1989). The level crossings are removed by a small amount of disorder.

\section{INFLUENCE OF COULOMB REPULSION ON THE AHARONOV-BOHM EFFECT}

As discussed for a non-interacting electron gas by Van Wees et al. (1989) and by Sivan et al. (1989), Aharonov-Bohm oscillations result from resonant tunneling through the quantum dot via edge states circulating along the dot perimeter [see Fig. 3(a)]. The presence of circulating edge states makes it possible to have conductance oscillations with a well-defined periodicity $\Delta B$ in a dot, just as the usual Aharonov-Bohm effect in a ring. There is, however, a difference - which went unnoticed in these papers. In each period $\triangle B$ the number of states below a given energy increases by one in a quantum dot - but stays constant in a ring. As a result, the Aharonov-Bohm magnetoconductance oscillations of a quantum dot are 
accompanied by an increase of the charge of the dot by one elementary charge per period. That is of no consequence if the Coulomb repulsion of the electrons can be neglected, but becomes important if the dot has a small capacitance $C$ to the reservoirs, since then the electrostatic energy $e^{2} / C$ associated with the incremental charging by single electrons has to be taken into account.

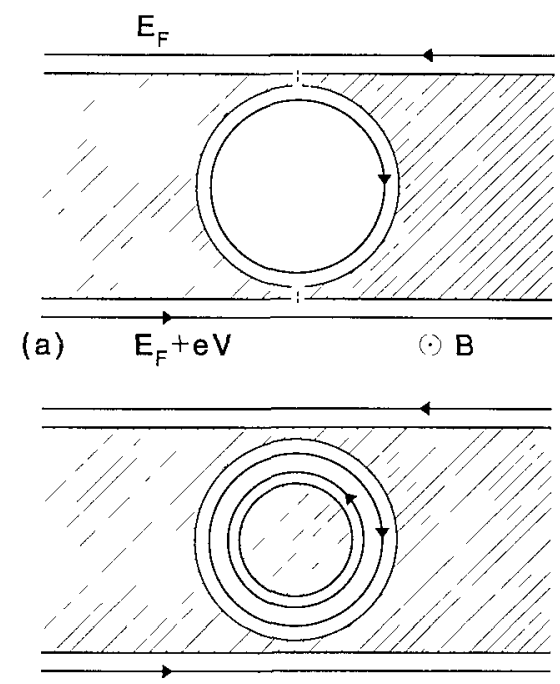

(b)

Fig. 3 (a) Quantum dot geometry. A gate (shaded) isolates a disc-shaped region in a two-dimensional electron gas from two reservoirs. Conduction through the dot occurs by tunneling (dashed lines), in the case of a small voltage difference $V$ between the reservoirs at equilibrium chemical potential $E_{\mathrm{F}}$. Edge states in the reservoirs and in the quantum dot are indicated, with arrows pointing into the direction of motion. (b) Geometry which can be transformed from a dot into a ring, by depleting the electron gas below the disc-shaped gate. Two sets of edge states, circulating in opposite directions, appear in a ring. [From Beenakker et al. (1990).]

To analyze this problem, we combine the results of the previous two sections. We apply (6) to the energy spectrum shown in Fig. 2. We consider here only the edge states from the lowest (spin-split) Landau level, so that the Aharonov-Bohm oscillations have a single periodicity. This corresponds to the strong-magnetic field limit. The magnetic field dependence of the edge states can be described approximately by a sequence of equidistant parallel lines,

$$
E_{p}=\text { constant }-\frac{\Delta E}{\Delta B}(B-p \Delta B),
$$

see Fig. 2. Sivan and Imry (1988) estimate, for a circular quantum dot of radius $R, \Delta \mathrm{B} \approx h / e A$ and $\Delta E \approx \hbar \omega_{\mathrm{c}} l_{\mathrm{m}} / 2 R$. These are order of magnitude estimates for a hard-wall confining potential. ${ }^{4}$ On substitution of (12) into (6), one finds the condition

\footnotetext{
4 For a smooth confining potential $V(r)$ (with $l_{\mathrm{m}} V^{\prime} \leq \hbar \omega_{\mathrm{c}}$ ) one has instead the cstimates $\Delta B=(h / e)[A(B)+$ $\left.B A^{\prime}(B)\right]^{-1}=(h / e A)\left[1-\hbar \omega_{\mathrm{c}} / R V^{\prime}(R)\right]^{-1}$ (Van Wecs et al, 1989), and $\Delta E=h / \tau=l_{\mathrm{m}}^{2} V^{\prime}(R) / R$, wherc $A(B)$ is the area cnclosed by the equipotential of radius $R$ at the guiding center encrgy $V(R)=E-\hbar \omega_{\mathrm{C}} / 2$ [Cf. (8) for $n=1$ ].
} 


$$
N\left(\Delta E+\frac{e^{2}}{C}\right)=\frac{\Delta E}{\Delta B} B+E_{\mathrm{F}}+\text { constant }
$$

for the location of the conductance peaks. The $B$-dependence of the reservoir Fermi energy can be neglected in (13) in the case of a hard-wall confining potential (since $d E_{\mathrm{F}} / d B \approx h \omega_{\mathrm{c}} / B<<$ $\Delta E / \Delta B$ ). The periodicity $\Delta B^{*}$ of the Aharonov-Bohm oscillations is thus given by 5

$$
\Delta B^{*}=\Delta B\left(1+\frac{e^{2}}{C \Delta E}\right) .
$$

We conclude from (14) that the charging energy enhances the spacing of two subsequent peaks in $G$ versus $B$ by a factor $1+e^{2} / C \Delta E$. The effect of the charging energy on the amplitude of the peaks is beyond the present analysis, but we surmise that the increase of the effective level spacing by an amount $e^{2} / C$ will lead to a larger peak amplitude at a given temperature. The periodicity of the magnetoconductance oscillations is lost if $\Delta B^{*}$ becomes so large that the linear approximation (12) for $E_{p}(B)$ breaks down. Since (12) holds at most over an energy range of the Landau level separation $\hbar \omega_{c}$, this suppression of the Aharonov-Bohm effect occurs when $(\Delta E / \Delta B) \Delta B^{*} \geq \hbar \omega_{c}$, i.e. when $e^{2} / C \geq \hbar \omega_{c}$.
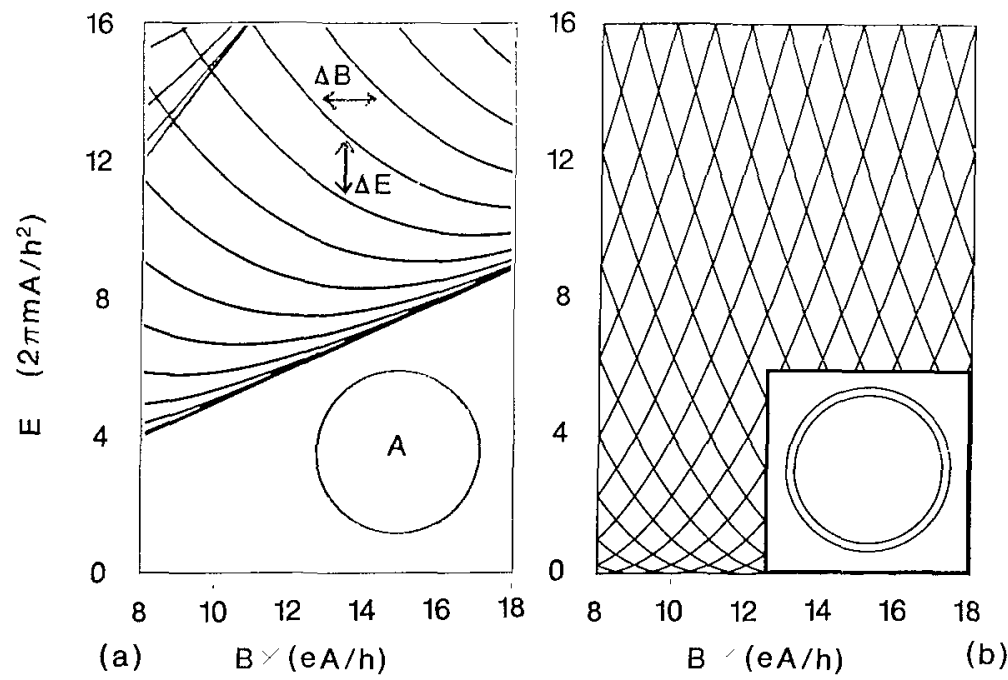

Fig. 4 Comparison of the energy levels in a disc and a ring. (a) Circular hardwall disc (Geerinckx et al. 1990). (b) Circular channel or ring of width $W \ll l_{\mathrm{m}}$ (Büttiker et al., 1983). The levels in (b) are plotted relative to the energy of the bottom of the one-dimensional subband in the channel. The case $W^{\prime} \geq l_{\mathrm{m}}$ is qualitatively the same as long as $S$ « $A$ (see Fig. 6). [From Beenakker et al. (1990).]

\footnotetext{
The cstimate for $\Delta E$ results from the correspondence between the level spacing and the period $\tau$ of the classical motion along the cquipotential, with guiding-center-drift velocity $V^{\prime}(R) / e B$.

5 In the case of a smooth confining potential, the term $\triangle B$ in the enhancement factor of (14) should be replaced by the term $\Delta B\left[1+(\Delta B / \Delta E)\left(d E_{F} / d B\right)\right]^{-1}=h / e A$, under the assumption that the Fermi energy in the rescrvoir is pinned to the lowest Landau level, i.c. $E_{\mathrm{F}}=\frac{1}{2} \hbar \omega_{\mathrm{c}}$
} 
The Aharonov-Bohm oscillations with bare periodicity $\Delta B=h / e A$ are recovered if one makes a hole in the disc, which is sufficiently large that the area $S$ of the conducting region is much smaller than the enclosed area $A$. The inner perimeter of the resulting ring supports a second set of edge states, which travel around the ring in the opposite direction as the first set of edge states at the outer perimeter [Fig. 3(b)]. We compare in Fig. 4 the energy spectrum for a disc and a ring. The two sets of clockwise and counter-clockwise propagating edge states in a ring are distinguished by the opposite sign of $d E_{p} / d B$, i.e. of the magnetic moment. Each set of edge states leads to oscillations in the magnetoconductance of a ring with the same period $\Delta B$, but shifted in phase (and in general with different amplitude, because the edge states at the inner perimeter have a smaller tunneling probability to the reservoir than those at the outer perimeter). The charging energy does not modify $\triangle B$ in a ring, because

$$
E_{p}(B)=E_{p}(B+\Delta B) \text { (ring). }
$$

In a disc, in contrast, one has according to (12),

$$
E_{p}(B)=E_{p+1}(B+\Delta B)(\text { disc })
$$

To illustrate the difference, we compare in Fig. 5 for disc and ring the renormalized energy levels $E_{p}^{*}$ [defined in (6)]. The effect of the charging energy in a ring is to open an energy gap of magnitude $e^{2} / C$ in $E_{p}^{*}$. This gap will affect the periodicity of the conductance oscillations as a function of $E_{\mathrm{F}}$, but not as a function of $B$.

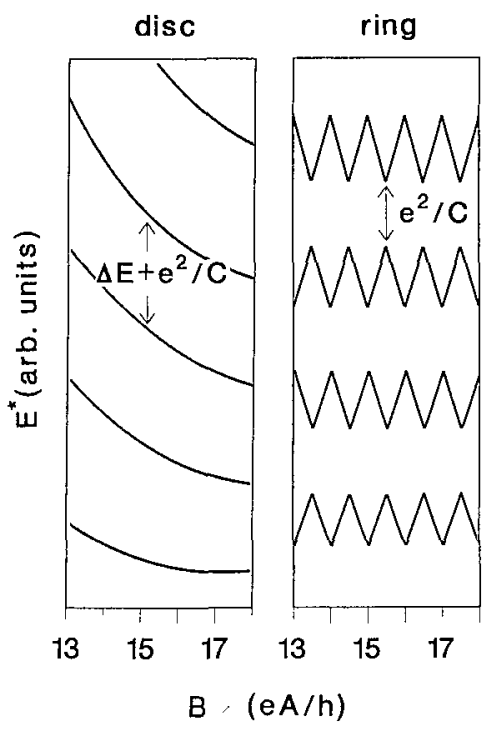

Fig. 5 Renormalized energy levels, defined by Eq. (6), corresponding to the bare energy levels shown in Fig. 4. Left panel: Disc geometry; Right panel: Ring geometry (the cusps will be rounded by a small amount of disorder).

\section{SUGGESTED EXPERIMENT}

A controlled experimental demonstration of the influence of Coulomb repulsion on the Aharonov-Bohm effect may be obtained in a system which can be transformed from a dot into a ring. What we have in mind is a geometry such as shown in Fig. 3(b), which has an additional disc-shaped gate within the gate of Fig. 3(a). By applying a negative voltage between this additional gate and the reservoir, one depletes the central region of the quantum dot, thereby transforming it into a ring. The capacitance $C$ between dot and reservoir is 
dominated by the capacitance between the dot and the disc-shaped gate, $C \approx \varepsilon A / d$, with $\varepsilon$ the dielectric constant of the material and $d$ the separation of the two-dimensional electron gas and the gate. Using the estimate $\Delta E=\hbar \omega_{\mathrm{c}} l_{\mathrm{m}} / 2 R$, and the parameters $\varepsilon=13 \varepsilon_{0}, m=0.07 m_{\mathrm{e}}, d=$ $50 \mathrm{~nm}$ appropriate for a GaAs-(Al,Ga)As heterostructure, one finds $e^{2} / C \Delta E \approx 10^{-6}$ $(\mathrm{m} / R)(\mathrm{T} / B)^{1 / 2}$. For a dot radius $R$ of $1 \mu \mathrm{m}$, and a magnetic field $B$ of a few Tesla, the charging energy is thus of the same magnitude as the level spacing of the edge states, so that a frequency doubling of the Aharonov-Bohm oscillations should be observable on depletion of the central region of the dot.

The area of the depleted central region should be sufficiently large that $S$ « $A$ (where $S$ is the conducting area of the ring). This ensures that a field increment $\Delta B=h / e A$ does not change the Landau level degeneracy $B e S / h$, since $\triangle B e S / h=S / A \ll 1$. In that case one has approximately $E_{p}(B)=E p(B+\Delta B)$, so that the Aharonov-Bohm oscillations recover the bare periodicity $\triangle B$ - even though the capacitance has become much smaller by depletion of the central region of the dot.

The case $S \sim A$, intermediate between a dot and a ring, is also of interest. In Fig. 6(a) we illustrate the single-electron energy levels $E_{p}$ and in Fig. $6(\mathrm{~b})$ the renormalized levels $E_{p}^{*} \equiv$ $E_{p}+(p-(1 / 2)) e^{2} / C$, for such a case. The Aharonov-Bohm oscillations of the magnetoconductance now have the bare periodicity $\Delta B$, but over a limited magnetic field range only.

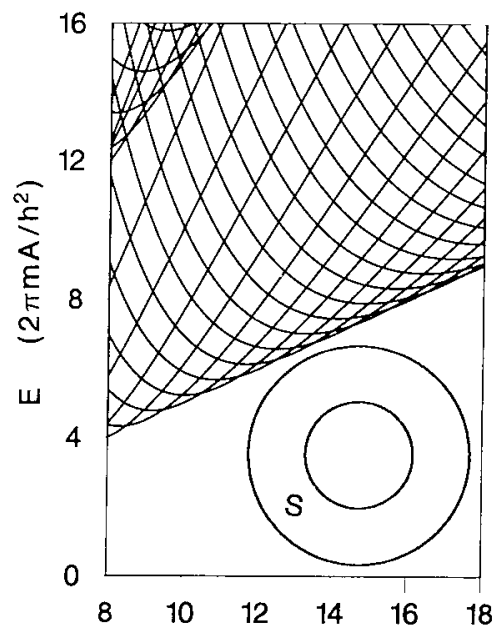

(a)

$$
\text { B }(e A / h)
$$

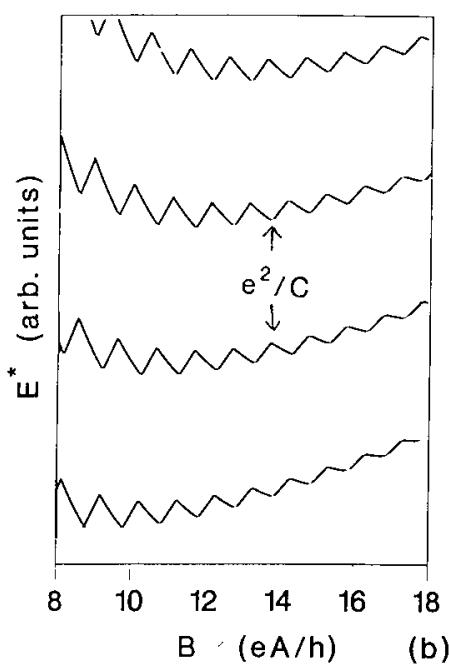

Fig. 6 (a) Approximate energy levels [according to (11)] as a function of magnetic field in a relatively wide ring, defined by $V(r)=m \omega_{0}^{2}\left(r-r_{0}\right)^{2} / 2$ with $\omega_{0} m A / h=10$. The area $A$ is defined by $A=\pi r$. The inset shows the region (of area $S$ ) which is accessible classically by electrons in the energy range shown in the figure. (b) Corresponding renormalized energy levels.

\section{ACKNOWLEDGMENTS}

We gratefully acknowledge the stimulating support of M. F. H. Schuurmans, and helpful discussions with $\mathrm{S}$. Colak on the electrostatic aspects of the problem. 


\section{REFERENCES}

Aharonov, Y., and Bohm, D., 1959, Significance of electromagnetic potentials in the quantum theory, Phys. Rev., 115:485.

Aharonov, Y., and Bohm, D., 1961, Further considerations on electro-magnetic potentials in the quantum theory, Phys. Rev., 123:1511.

Amman, M., Mullen, K., and Ben-Jacob, E., 1989, The charge-effect transistor, J. Appl. Phys., 65:339.

- Aronov, A. G., and Sharvin, Yu. V., 1987, Magnetic flux effects in disordered conductors, Rev. Mod. Phys., 59:755.

Averin, D. V., and Korotkov, A. N., Correlated single-electron tunneling via mesoscopic metal particle: Effects of the energy quantization, Zh. Eksp. Teor. Fiz. [Sov. Phys. $J E T P]$ (to be published).

Beenakker, C. W. J., Van Houten, H., and Staring, A. A. M., 1990, Influence of Coulomb repulsion on the Aharonov-Bohm effect in a quantum dot, submitted to Phys. Rev. Lett.

Beenakker, C. W. J., and Van Houten, H., 1991, Quantum transport in semiconductor nanostructures, in "Solid State Physics," edited by H. Ehrenreich and D. Turnbull, Academic Press, New York.

Bogachek, E. N., and Gogadze, G. A., 1973, Oscillation effects of the "flux quantization" type in normal metals, Zh. Eksp. Teor. Fiz., 63:1839 [Sov. Phys. JETP, 36:973].

Brandt, N. B., Gitsu, D. V., Nikolaeva, A. A., Ponomarev, Ya. G., 1977, Investigation of size effects in thin cylindrical bismuth single crystals in a magnetic field, Zh. Eksp. Teor. Fiz., 72:2332 [Sov. Phys. JETP, 45:1226].

Bryant, G. W., 1987, Electronic structure of ultrasmall quantum-well boxes, Phys. Rev. Lett., $59: 1140$.

Büttiker, M., Imry, Y., and Landauer, R., 1983, Josephson behavior in small normal onedimensional rings, Phys. Lett. A, 96:365.

Büttiker, M., 1987, Zero-current persistent potential drop across small-capacitance Josephson junctions, Phys. Rev. B, 36:3548.

Darwin, C. G., 1930, The diamagnetism of the free electron, Proc. Camb. Phil. Soc., 27:86.

Fock, V., 1928, Bemerkung zur Quantelung des harmonischen Oszillators im Magnetfeld, Z. Phys., 47:446.

Geerinckx, F., Peeters, F. M., and Devreese, J. T., 1990, Effect of the confining potential on the rnagneto-optical spectrum of a quantum dot, J. Appl. Phys. (to be published).

Glazman, L. I., and Shekhter, R. I., 1989, Coulomb oscillations of the conductance in a laterally confined heterostructure, J. Phys. Condens. Matter, 1:5811.

Gorter, C. J., 1951, A possible explanation of the increase of the electrical resistance of thin metal films at low temperatures and small field strengths, Physica, 17:777.

Korotkov, A. N., Averin, D. V., and Likharev, K. K., 1990, Single-electron charging of the quantum wells and dots, Physica B, 165\&166:927.

Kouwenhoven, L. P., Van Wees, B. J., Van Der Enden, B., Harmans, C. J. P. M., and Timmering, C. E., 1990, Electronic transport through single and multiple quantum dots: The formation of a 1D crystal bandstructure, in "Proc. 20th Int. Conf. on the Physics of Semiconductors," edited by J. Joannopoulos, World Scientific, London.

Kumar, A., Laux, S. E., and Stern, F., 1990, Electron states in a GaAs quantum dot in a magnetic field, Phys. Rev. B, 42:5166.

Laux, S. E., Frank, D. J., and Stern, F., 1988, Quasi-one-dimensional electron states in a split-gate GaAs/AlGaAs heterostructure, Surf. Sci., 196:101.

Likharev, K. K., 1988, Correlated discrete transfer of single electrons in ultrasmall tunnel junctions, IBM J. Res. Dev., 32:144.

Maksym, P. A., and Chakraborty, T., 1990, Quantum dots in a magnetic field: Role of electron-electron interactions, Phys. Rev. Lett., 65:108.

Meirav, U., Kastner, M. A., Heiblum, M., and Wind, S. J., 1989, A one-dimensional electron gas in GaAs: Periodic conductance oscillations as a function of density, Phys. Rev. B, 40:5871. 
Meirav, U., Kastner, M. A., and Wind, S. J.,1990, Single-electron charging and periodic conductance resonances in GaAs nanostructures, Phys. Rev. Lett., 65:771.

Scott-Thomas, J. H. F., Field, S. B., Kastner, M. A., Smith, H. I., and Antoniadis, D. A., 1989 , Conductance oscillations periodic in the density of a one-dimensional electron gas, Phys. Rev. Lett., 62:583.

Sivan, U., and Imry, Y., 1988, De Haas-Van Alphen and Aharonov-Bohm-type persistent current oscillations in singly connected quantum dots, Phys. Rev. Lett., 61:1001.

Sivan, U., Imry, Y., and Hartzstein, C., 1989, Aharonov-Bohm and quantum Hall effects in singly connected quantum dots, Phys. Rev. B, 39:1242.

Staring, A. A. M., Van Houten, H., Beenakker, C. W. J., and Foxon, C. T., 1990, Coulomb-regulated conductance oscillations in a disordered quantum wire, in "High Magnetic Fields in Semiconductor Physics III," edited by G. Landwehr, SpringerVerlag, Berlin.

Van Houten, H., and Beenakker, C. W. J., 1989, "Comment on "Conductance oscillations periodic in the density of a one-dimensional electron gas'," Phys. Rev. Lett., 63:1893.

Van Loosdrecht, P. H. M., Beenakker, C. W. J., Van Houten, H., Williamson, J. G., Van Wees, B. J., Mooij, J. E., Foxon, C. T., and Harris, J. J., 1988, Aharonov-Bohm effect in a singly connected point contact, Phys, Rev. B, 38:10162.

Van Wees, B. J., Kouwenhoven, L. P., Harmans, C. J. P. M., Williamson, J. G., Timmering, C. E., Broekaart, M. E. I., Foxon, C. T., and Harris, J. J., 1989, Observation of zero-dimensional states in a one-dimensional electron interferometer, Phys. Rev. Lett., 62:2523.

Washburn, S., and Webb, R. A., 1986, Aharonov-Bohm effect in normal metal: Quantum coherence and transport, Adv. Phys, 35:375.

Wingreen, N. S., and Lee, P. A., 1990, oral presentation at the NATO Adv. Study Inst. on Quantum Coherence in Mesoscopic Systems. 